\title{
The effect of curcumin on consolidation and retrieval memory following unilateral labyrinthectomy
}

\author{
Zahra Lamiyan, Amin Abdollahazade Fard, Naser Khalaji \\ Department of Physiology, Faculty of Medicine, Urmia University of Medical Sciences, Urmia, Iran
}

\begin{abstract}
Objectives. Nowadays, vestibular system disorders are common. The aim of this study was to evaluate the effect of curcumin on Vestibular compensation (VC) and behavioral changes following unilateral labyrinthectomy in male rats. Methods. Forty adult male Wistar rats were randomly divided into 5 groups: control; without labyrinthectomy and injection of curcumin with passive avoidance learning and memory retrieval testing in 3,6 and 10 days after learning, group 2; labyrinthectomy with passive avoidance learning and memory retrieval testing, group 3; labyrinthectomy with passive avoidance learning and injection of curcumin then memory retrieval testing, group 4; passive avoidance learning, labyrinthectomy and memory retrieval testing and group 5; passive avoidance learning, labyrinthectomy with injection of curcumin and memory retrieval testing.

The rats were anesthetized at the end of behavior experiment. The brain tissue was removed and brain stem was taken for morphological assessment.

Outcomes. The results of this study showed that labyrinthectomy significantly reduced the latency of entering the dark section of the shuttle box compared to the other groups on the 6th and 10th day learning $(P<0.05)$. In the curcumin groups with labyrinthectomy (groups 3 and 5), learning which was performed before and after labyrinthectomy, the latency of the entrance to the dark section was decreased but not significant in comparison to the control group $(P>0.05)$. In group 4, after learning and then labyrinthectomy, the latency of the entering the dark section was decreased but not significant in comparison to the control group $(P>0.05)$.

In the morphological assessment, in the labyrinthectomy group, the increase of supportive cells in the brain stem and hippocampus was observed in comparison with the control group and also deformation and decrease of neurons in the brain stem was observed, but it was not significant change in curcumin group compared to the control group.

Conclusion. Unilateral labyrinthectomy caused behavioral disorders and also tissue changes of brain stem on labyrinthectomy side. curcumin had a protective effect on the nervous system maybe by neurotropic, anti-inflammatory and anti-apoptotic properties.
\end{abstract}

Keywords: vestibular compensation, unilateral labyrinthectomy, vestibular system, learning and memory, curcumin

\section{INTRODUCTION}

Vestibular disorders are common and often disabling. Unilateral labyrinthectomy (UL) causes a syndrome of oculomotor, postural and sensory disorders, which diminish over time in a process of behavioural recovery known as vestibular compensation (VC) $(1,2)$. The vestibular system is the sensory system that contributes to the sense of balance and spatial orientation as well as stabilizes gaze for head velocities and frequencies in the range of natural head movements for the purpose of coordinat- ing movement with balance (3). The results of some studies have shown that Collateral sprouting occurs following UL in amphibians (4), although it does not occur in verte- brates brainstem at any post-lesional time (5). The findings from one of our previous study showed that the dieters neuron degeneration in ipsilateral occurs after UL (6). Herbal medicine has been commonly used over the years for treatment and prevention of diseases. One of them is curcumin, the main active ingredient in turmeric (7). Due to the phenolic group in curcumin molecular structure, it has antioxidant effects $(8,9)$. 
Oral administration of curcumin significantly reduces lipid peroxidation and increases glutathione in the brain which is considered as a central nervous system protector (10). Curcumin improves cognitive impairment and increases the level of acetylcholine in the brain cortex of diabetic rats (11). Destruction of the vestibular system causes a person to experience visual and motor disorders. curcumin plays a role in processes such as cell proliferation, differentiation and cell migration (12) and is highly effective in preventing the degeneration of neuroglial support cells due to its anti-apoptotic effects (13). Therefore, this study examines the protective effects of curcumin in $\mathrm{VC}$ and the study of behavioral changes following UL.

\section{MATERIALS AND METHODS}

\section{Study design}

Experiments were carried out on 40 adult male Wistar rats $(230 \pm 30 \mathrm{~g}$ body weight $)$. The animals were purchased from the animal house of Urmia University of Medical sciences and kept in standard condition (12h light/dark cycle and $22 \pm 2$ temperature) and ad libitum feeding. The animals were randomly divided into 5 groups; control or 1(without unilateral labyrinthectomy and curcumin, only injection of $0.2 \mathrm{ml}$ ethyl oleate, IP, every day for 15 days and passive avoidance learning with shuttle box then memory retrieval testing on 3, 6 and 10 days after learning), 2 (the next day after unilateral labyrinthectomy, $0.2 \mathrm{ml}$ ethyl oleate, IP, every day for 15 days and Passive avoidance learning with shuttle box then Memory retrieval testing on 3, 6 and 10 days after learning), 3 (the next day after unilateral labyrinthectomy, $20 \mu \mathrm{mol} / \mathrm{kg}$, IP every day for 15 days and passive avoidance learning with shuttle box then memory retrieval testing on 3, 6 and 10 days after learning), 4 (the next day after Passive avoidance learning unilateral labyrinthectomy, $0.2 \mathrm{ml}$ ethyl oleate, IP, every day for 15 days and memory retrieval testing on 3, 6 and 10 days after learning), 5 (the next day after Passive avoidance learning unilateral labyrinthectomy, $20 \mu \mathrm{mol} /$ $\mathrm{kg}$, IP every day for 15 days and memory retrieval testing on 3, 6 and 10 days after learning). All animal experiments were performed according to the instructions of the Urmia University Medical Sciences Ethics Committee.

\section{Unilateral labyrinthectomy}

Animals were anesthetized with pentobarbital sodium $(40 \mathrm{mg} / \mathrm{kg}$, ip). Then unilateral labyrinthectomy was performed by electro coagulation apparatus that composed two electrodes (cathode and anode), the cathode was inserted into inner ear and the anode was connected to skin with $20 \mathrm{~m} \mathrm{AM}$ voltage for 1 minute. To reduce pain, we can make $10 \mathrm{~mA}$ for 2 minutes. After labyrinthectomy to prevent infection, tetracycline was applied to the site. Then the rats were controlled for proper labinterectomy. When we pull the rat from the tail, it bends towards the labyrinthectomy side.

\section{Shuttle-Box passive avoidance learning}

This device has two bright and dark compartments separated by a door. In avoidance training, a light $(60 \mathrm{~W})$ was switched on alternately in the two compartments and used as a conditioned stimulus (CS). The CS preceded the onset of the unconditioned stimulus (US) by $5 \mathrm{~s}$ and overlapped it for 25 s. In the dark chamber the shock $(0.1 \mathrm{~mA})$ as unconditioned stimulus (US) was applied continuously to the grid floor of the device.

At the start of learning test, the rats were placed individually on the right compartment of a shuttle box. Then the lamp in the compartment was turned on and 10 seconds later the door between the two compartments was opened. The rat moves between the two compartments for 10 minutes to get used to the environment. The steps mentioned above were repeated 30 minutes later. As soon as the rat entered the dark chamber, the door was closed and an electric shock was delivered to the floor at $50 \mathrm{~Hz}, 1 \mathrm{~mA}$ for 2 seconds. After using the shock, the door opens and the rat moves from the dark chamber to the light chamber quickly. Then the animal removes it from the compartment and two minutes later, passive avoidance learning was repeated. Learning is done if the rat does not enter dark compartment during 120 seconds.

\section{Shuttle-Box retrieval and memory consolidation test}

To perform a memory retrieval and memory consolidation of passive learning avoidance, the rats were individually placed in a light chamber. Immediately after placement of the rat, the lamp 
turned on and the door was opened 10 seconds later. The time until arrival in the dark chamber was measured and recorded by a chronometer. This time was followed up to 600 seconds. The rats that did not enter the dark chamber within 600 seconds had a latency time of 600 seconds and Memory consolidation was occurred.

\section{Histological study}

Hematoxylin and eosin (H\&E)-stained brain sections from all studied groups were prepared for histological studies.

\section{Statistical analysis}

Data were expressed as the mean \pm SD. Statistical significance of differences was assessed with one-way ANOVA followed by Tukeys' test. $\mathrm{P} \leq$ 0.05 was considered as statistically significant.

\section{RESULTS}

\section{Learning time at first day}

The results showed that the mean of learning time was the same in all groups and was 120 minute. The frequency of shock was also in all groups once. There are no significant differences between study groups (Figure 1).

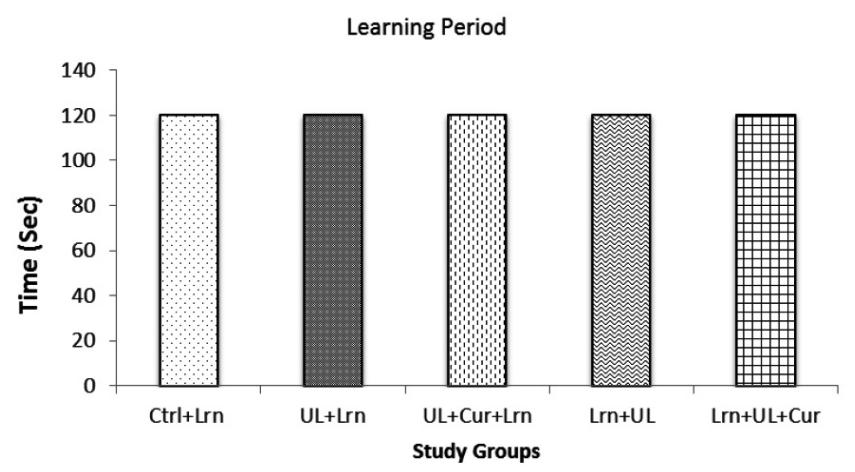

FIGURE 1. Comparison of learning time and memory consolidation at the start on study Ctrl: Control, UL:unilateral labirentectomy, Cur:Curcumin, Lrn: Learning

\section{Memory retrieval $48 \mathrm{~h}$ after learning}

Average latency in entering the dark chamber $48 \mathrm{~h}$ after was $600,451 \pm 268,528 \pm 133,600$ and $555 \pm 83$ minute in Control ( Ctrl), Unilateral Labyrinthectomy + learning (UL+Lrn), Unilateral Labyrinthectomy + Curcumin + learning $(\mathrm{UL}+\mathrm{Cur}+\mathrm{L}-$ rn), learning + Unilateral Labyrinthectomy $(\mathrm{Lrn}+\mathrm{UL})$ and learning + Unilateral Labyrinthecto- my + Curcumin (Lrn+UL+Cur) groups respectively. The results indicate a decrease in the average delay time in the second group that was labyrinthectomized and then was learned in comparison with control and other groups. But this decrease in time of intering was not significant ( $\mathrm{p}=0.2$, Figure 2 ).

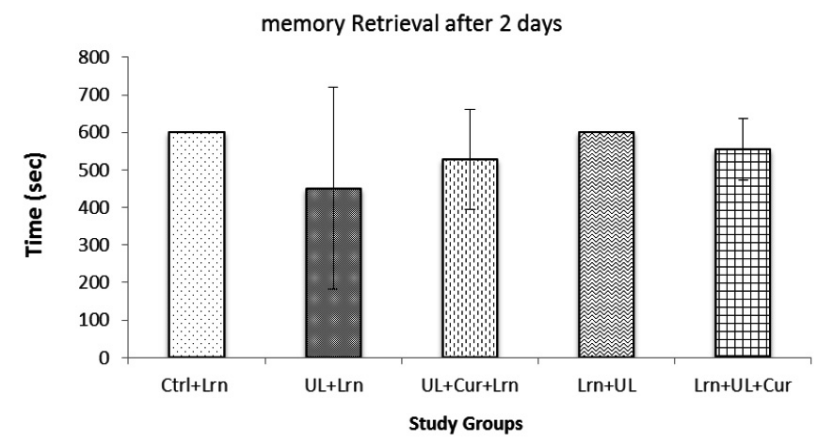

FIGURE 2. Means $\pm S D$ of memory retrieval 2 day after learning Ctrl: Control, UL:unilateral labirentectomy, Cur:Curcumin, Lrn: Learning, data shows as means \pm $S D$

\section{Memory retrieval 5 day after learning}

Average latency in entering the dark chamber 5 day after was $600,161 \pm 151,471 \pm 240,485 \pm 264$ and $473 \pm 236$ minute in Control (Ctrl), Unilateral Labyrinthectomy + learning (UL + Lrn), Unilateral Labyrinthectomy + Curcumin + learning (UL+Cur+Lrn), learning + Unilateral Labyrinthectomy (Lrn+UL) and learning + Unilateral Labyrinthectomy + Curcumin (Lrn+UL+Cur) groups respectively. The results show a significance decrease in the average delay time in the second group that was labyrinthectomized and then was learned in comparison with control $(p<0.01)$ If it was not significant in comparison to other groups (Figure 3 ).

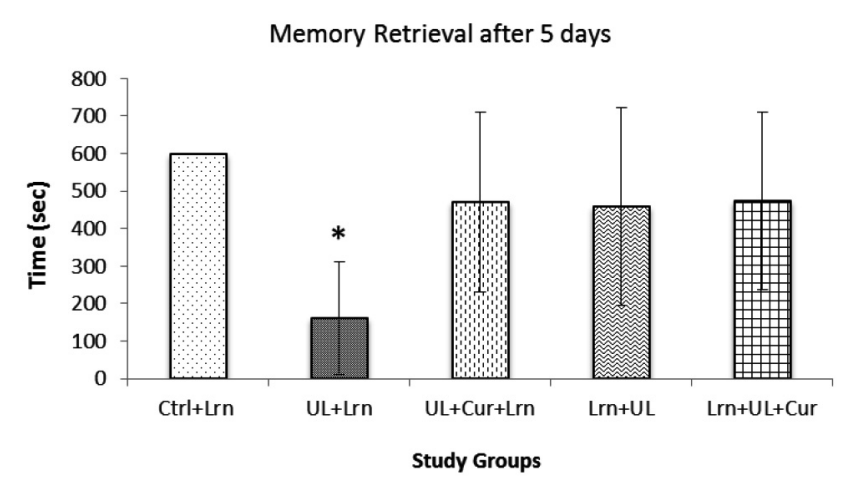

FIGURE 3. Means $\pm S D$ of memory retrieval after 5 day after learning, Ctrl: Control, UL:unilateral labirentectomy, Cur:Curcumin, Lrn: Learning. * shows the signaficancy whit other groups $(p \leq 0.05)$ 


\section{Memory retrieval 9 day after learning}

Average latency in entering the dark chamber 9day after was $600,41 \pm 29,459 \pm 261,458 \pm 264$ and $573 \pm 50$ minute in Control (Ctrl), Unilateral Labyrinthectomy + learning (UL+Lrn), Unilateral Labyrinthectomy + Curcumin + learning $(\mathrm{UL}+\mathrm{Cur}+\mathrm{L}-$ rn), learning + Unilateral Labyrinthectomy $($ Lrn+UL) and learning +Unilateral Labyrinthectomy + Curcumin (Lrn+UL+Cur) groups respectively. The results show a significance decrease in the average delay time in the second group that was labyrinthectomized and then was learned in comparison with other studied groups $(\mathrm{p}<0.001)$ (Figure 4 and Figure 5).

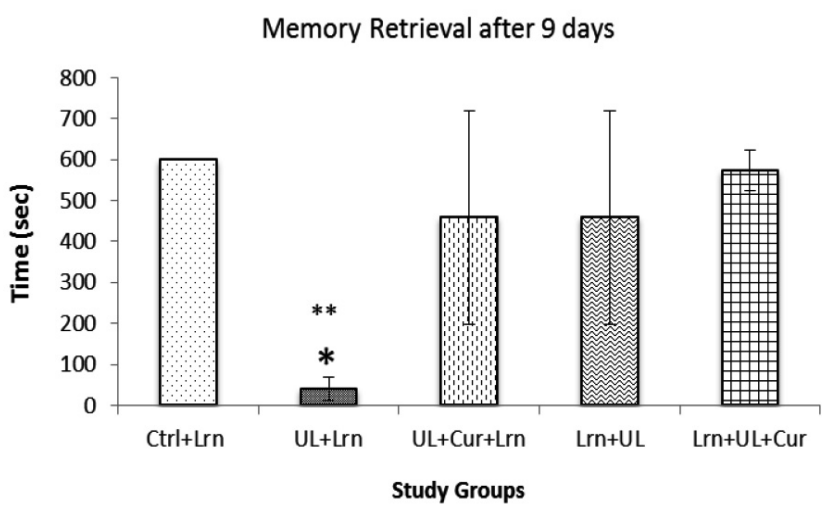

FIGURE 4. Means $\pm S D$ of memory retrieval after 9 day after learning, Ctrl: Control, UL:unilateral labirentectomy, Cur:Curcumin, Lrn: Learning, * shows the signaficancy whit other groups ( $p \leq 0.05)$

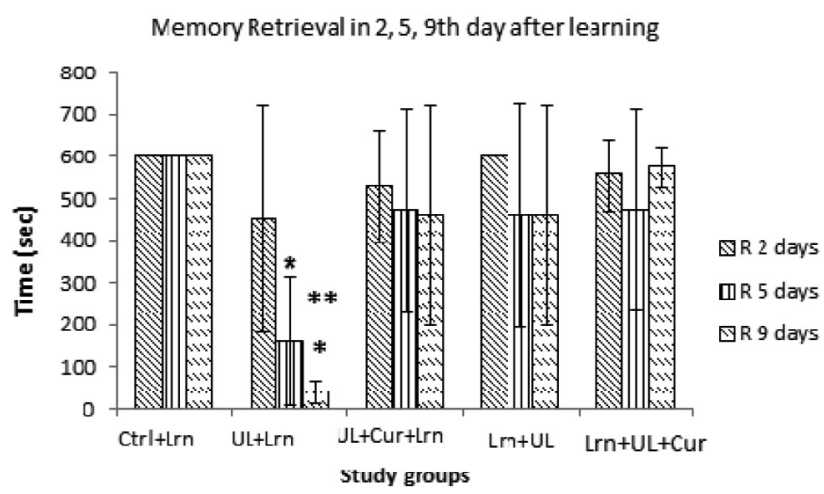

FIGURE 5. Comparison of retrieval memory at 3, 6, and 10th of study period, Ctrl: Control, UL:unilateral labirentectomy, Cur:Curcumin, Lrn: Learning, * shows the signaficancy whit other days ( $p \leq 0.05)$

\section{Vestibular nucleus histological changes}

The morphological findings of vestibular nucleus neurons in the control group after staining with H\&E showed that the shape of the neurons was dis- tinct and polygonal and also nucleus of the cells and long dendrites was visible. The high number of neurons, as well as low neuroglial cells, was evident (Figure $6 \mathrm{~A}$ and $\mathrm{B}$ ). But after unilateral labyrenthectomy, at the side of damage, vestibular nucleus neurons were degenerated and the number of neurons decreased. Also, the dendrites were shorter and the size of the cells was smaller in comparison with control. But neurogilia had increased (Figure $6 \mathrm{D})$. While on the healthy side of the same group with no labyrinthectomy, Neurons were healthy and the number of neurogilia was higher than that of the control group but the neuroglia was less than the side of the damage, and some cells appeared small in size (Figure $6 \mathrm{C}$ ). As shown in figure $6 \mathrm{E}$ and $\mathrm{F}$ curcumin administration reduced the damage of vestibular nucleus.

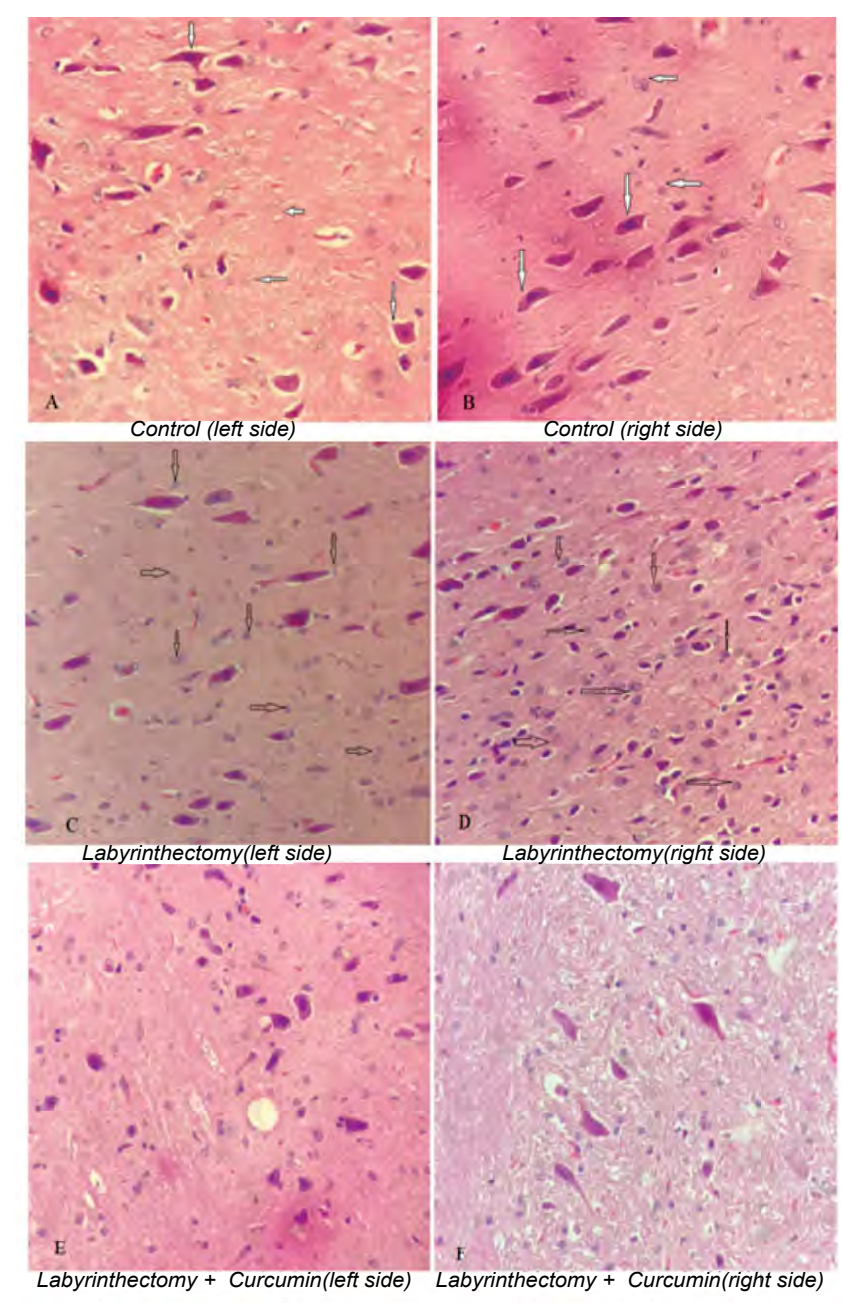

FIGURE 6. Photomicrograph of right and left vestibular nucleus (H\&E staining). A and $B$ are control groups (left and right side respectively), $C$ and $D$ labyrinthectomy group, $E$ and $F$ are curcumin treated group. Arrows show the polygonal and microglia cells (×400). 


\section{DISCUSSION}

The results of this study showed that unilateral labyrinthectomy impairs learning and memory recall. The vestibular system plays a significant role in the production of situational, ocular and motor reflexes. It also plays a role in memory and even recognition (3) vestibular disorders not only causes reflexes impairment but also causes anxiety, memory and attention disorders $(14,15)$. The results of present study, in accordance with previous studies, showed that destruction of the inner ear caused impairment causes impairment in memory consolidation and retrieval. Our results showed that the memory and retrieval process was impaired on the 6th and 10th day after labyrinthectomy. While, if learning was initially done, memory and retrieval impairment caused by laptomentomy will be less. This finding confirms the vestibular systems role in the onset of the learning process. These findings confirm the results of previous studies on the role of the vestibular system in the learning process (15). The results of the present study showed that there was no memory retrieval impairment when using curcumin following lacrerectomy. SoukhakLari et al. investigated the effect of curcumin on the passive avoidance learning test, and found that administration of curcumin Improved memory (16).
The results of this study confirm the results of previous studies that emphasize the role of chroquine on memory improvement $(17,18)$. The effects of memory recovery following treatment with curcumin may be due to anti-inflammatory properties of curmumin (19) or antioxidant effects and neuroprotection of this agent $(17,20,21)$. In present study, the protective effect of curcumin on vestibular nucleus cells is evident. It confirms the results of previous studies (22).

\section{CONCLUSION}

Finally, the results of this study showed that unilateral labyrintectomy causes learning process disruption, particularly when labyrinthectomy was occurred before learning. Also, the results showed curcumin has a protective effect on the learning process and memory retrieval after labyrinthectomy. This effect can be due to its anti-oxidant, anti-inflammatory properties and neuroprotective properties.

\section{Acknowledgment}

This article is a part on MSc student thesis of medical physiology and also supported by Urmia University of Medical Sciences (grant No: 2114).

Conflict of interest: none declared

\section{REFERENCES}

1. Smith PF, Curthoys IS. Mechanisms of recovery following unilateral labyrinthectomy: a review. Brain Research Reviews. 1989;14(2):15580.

2. Curthoys IS. Vestibular compensation and substitution. Current Opinion in Neurology. 2000;13(1):27-30.

3. Lacour M, Bernard-Demanze L. Interaction between vestibular compensation mechanisms and vestibular rehabilitation therapy: 10 recommendations for optimal functional recovery. Frontiers in Neurology. 2015;5:285.

4. Dieringer N. â€ Vestibular compensationâ€ $€^{\mathrm{TM}}$ : neural plasticity and its relations to functional recovery after labyrinthine lesions in frogs and other vertebrates. Progress in Neurobiology. 1995;46(2-3):97129.

5. Gacek RR, Lyon MJ, Schoonmaker J. Ultrastructural changes in vestibulo-ocular neurons following vestibular neurectomy in the cat. Annals of Otology, Rhinology \& Laryngology. 1988;97(1):42-51.

6. Khalaji N, Sarkisian V, Sarkissian J. The effect of central Asian cobra venom on the vestibular compensation and regeneration of neuron. The Journal of Urmia University of Medical Sciences. 2013; 24(9):689-701.

7. Gomez-Pinilla $F$. The influences of diet and exercise on mental health through hormesis. Ageing Research Reviews. 2008;7(1):49-62.

8. Garcia Alloza M, Borrelli L, Rozkalne A, Hyman B, Bacskai B. Curcumin labels amyloid pathology in vivo, disrupts existing plaques, and partially restores distorted neurites in an Alzheimer mouse model. Journal of Neurochemistry. 2007;102(4):1095-104.

9. Khalaji N, Zeinali A, Purjabali M, Bolurani K, Fard AA. The Effect of Bioactive Component of Turmeric (curcumin) on Liver ComplicationsInduced by Compact Fluorescent Lamps (CFLs) in Rats. Shiraz E-Medical Journal. 2018(In Press).

10. Rajakrishnan V, Viswanathan P, Rajasekharan K, Menon VP. Neuroprotective role of curcumin from Curcuma longa on ethanolâ€ induced brain damage. Phytotherapy Research. 1999;13(7):571-4.

11. Kuhad A, Chopra K. Curcumin attenuates diabetic encephalopathy in rats: behavioral and biochemical evidences. European Journal of Pharmacology. 2007;576(1-3):34-42.

12. Subramanian M, Devasagayam TP, Singh B. Diminution of singlet oxygen-induced DNA damage by curcmin and related antioxidants. Mutation Research/Fundamental and Molecular Mechanisms of Mutagenesis. 1994;311(2):249-55.

13. Tehranipour M, Khayatzadeh J, Javaherifard R. The protective effects of curcuma longa total extract on spinal cord neuroglia cell degeneration after the sciatic nerve compression in rats. Arak Medical University Journal 2010, Volume 13 , Number 1 (50); 83-89.

14. Smith $P$, Zheng Y. From ear to uncertainty: Vestibular contributions to cognitive function. Frontiers in Integrative Neuroscience. 2013;7:84.

15. Zheng Y, Mason-Parker SE, Logan B, Darlington CL, Smith PF, Abraham WC. Hippocampal synaptic transmission and LTP in vivo are intact following bilateral vestibular deafferentation in the rat. Hippocampus. 2010;20(4):461-8.

16. SoukhakLari R, Moezi L, Pirsalami F, Ashjazadeh N, Moosavi M. The passive avoidance memory improving effect of curcumin in young 
adult mice: Considering hippocampal MMP-2, MMP-9 and Akt/ GSK3î². PharmaNutrition. 2018;6(3):95-9.

17. Lim DW, Son HJ, Um MY, Kim I-H, Han D, Cho S, et al. Enhanced cognitive effects of demethoxycurcumin, a natural derivative of curcumin on scopolamine-induced memory impairment in mice. Molecules. 2016;21(8):1022.

18. Sarlak Z, Oryan S, Moghaddasi M. Interaction between the antioxidant activity of curcumin and cholinergic system on memory retention in adult male Wistar rats. Iranian journal of basic medical sciences. 2015;18(4):398.

19. Bengmark S. Curcumin, an atoxic antioxidant and natural NFkappaB, cyclooxygenase-2, lipooxygenase, and inducible nitric oxide synthase inhibitor: A shield against acute and chronic diseases.
Journal of Parenteral and Enteral Nutrition. 2006;30(1):45-51. JPEN J Parenter Enteral Nutr. 2006 Jan-Feb;30(1):45-51.

20. Ringman JM, Frautschy SA, Cole GM, Masterman DL, Cummings $\mathrm{JL}$. A potential role of the curry spice curcumin in Alzheimer's disease. Current Alzheimer Research. 2005;2(2):131-6.

21. Vajragupta $\mathrm{O}$, Boonchoong $\mathrm{P}$, Watanabe $\mathrm{H}$, Tohda $\mathrm{M}$, Kummasud $\mathrm{N}$, Sumanont $Y$. Manganese complexes of curcumin and its derivatives: Evaluation for the radical scavenging ability and neuroprotective activity. Free Radical Biology and Medicine. 2003;35(12):1632-44.

22. Tighilet B, Brezun JM, Dit Duflo Sylvie G, Gaubert Cl, Lacour M. New neurons in the vestibular nuclei complex after unilateral vestibular neurectomy in the adult cat. European Journal of Neuroscience. 2007;25(1):47-58. 